\title{
Hybrid operating strategies in the trade-off between fuel consumption and emissions
}

Sven Eberts, H.-J. Berner

FKFS

M. Bargende

FKFS/IVK, Universität Stuttgart

This manuscript is not available according to publishing restriction.

Thank you for your understanding. 